\title{
Myxome odontogène : présentation d'un cas clinique
}

\author{
Bellasfar $\mathbf{S}^{1}$, Khemira $\mathrm{A}^{2}$, Blouza $\mathbf{I}^{2}$, Turki $\mathrm{S}^{2}$ \\ ${ }^{1}$ Service de Stomatologie, Centre de Santé de Soins de base, Oued-Ellil, Tunisie \\ ${ }^{2}$ Service d'Odontologie, Hôpital militaire, Tunis, Tunisie \\ Mohamed.BenMoussa@fphm.rnu.tn
}

Le myxome odontogène est une tumeur bénigne rare, dérivée de la partie mésenchymateuse de l'appareil odontogénique et, du fait de la fréquence de son association à une dent incluse, de la portion mésenchymateuse de germe dentaire (Choi et al.2003).

Cette pathologie touche quasi exclusivement les maxillaires s'opposant ainsi au mysome des parties molles qui prédomine aux extrémités ; quelques cas exceptionnels ont été cependant décrits sur la gencive, dans les fosses nasales, les sinus, les glandes parotides... (Freedman et al.1992, Kawasaki et al. 1997).

Un cas de myxome odontogène est rapporté. Il s'agit d'un homme de 36 ans en bon état général, sans antécédents pathologiques. L'examen clinique révèle une tuméfaction sessile, de $1,5 \mathrm{~cm}$ de diamètre, siégeant sur la gencive dans la région de 11 et 21 . La lésion qui évolue depuis près de 4 mois a entrainé un déplacement des dents (malposition de la centrale supérieure gauche qui présente une légère rotation). Cette tuméfaction est couverte par une muqueuse saine : pas de saignement au contact; pas de douleurs à la palpation. Il n'y a pas d'adénopathies cervico-faciales. L'examen radiologique montre une lésion qui s'étend entre les racines des dents voisines. L'exérèse a été réalisée en une seule pièce par curetage du tissu osseux et du périoste qui a été laissé en place. Un traitement à base d'antibiotiques, d'antalgiques et de bains de bouche a été prescrit. L'examen anatomo-pathologique a montré des aspects histopathologiques et histochimiques concordant avec ceux du myxome. 